\title{
PDGFRA gene rearrangements are frequent genetic events in PDGFRA-amplified glioblastomas
}

\author{
Tatsuya Ozawa, ${ }^{1,2}$ Cameron W. Brennan, ${ }^{2,3,12}$ Lu Wang, ${ }^{4}$ Massimo Squatrito, ${ }^{1,2}$ Takashi Sasayama, ${ }^{5}$ \\ Mitsutoshi Nakada, ${ }^{6}$ Jason T. Huse, ${ }^{4}$ Alicia Pedraza, ${ }^{3}$ Satoshi Utsuki, ${ }^{7}$ Yoshie Yasui, $^{7}$ Adesh Tandon, ${ }^{8}$ \\ Elena I. Fomchenko, ${ }^{1,2}$ Hidehiro Oka, ${ }^{7}$ Ross L. Levine, ${ }^{9}$ Kiyotaka Fujii, ${ }^{7}$ Marc Ladanyi, ${ }^{4}$ \\ and Eric C. Holland ${ }^{1,2,10,11}$ \\ ${ }^{1}$ Department of Cancer Biology and Genetics, Memorial Sloan-Kettering Cancer Center, New York, New York 10065, USA; \\ ${ }^{2}$ Brain Tumor Center, Memorial Sloan-Kettering Cancer Center, New York, New York 10065, USA; ${ }^{3}$ Department of \\ Neurosurgery and Human Oncology and Pathogenesis Program, Memorial Sloan-Kettering Cancer Center, New York, New \\ York 10065, USA; ${ }^{4}$ Department of Pathology and Human Oncology, Pathogenesis Program, Memorial Sloan-Kettering Cancer \\ Center, New York, New York 10065, USA; ${ }^{5}$ Department of Neurosurgery, Kobe University Graduate School of Medicine, Kobe, \\ Hyogo 650-0017, Japan; ${ }^{6}$ Department of Neurosurgery, Graduate School of Medical Science, Kanazawa University, Kanazawa, \\ Ishikawa 920-8641, Japan; ${ }^{7}$ Department of Neurosurgery, Kitasato University School of Medicine, Sagamihara, Kanagawa \\ 252-0374, Japan; ${ }^{8}$ Department of Neurosurgery, The Albert Einstein College of Medicine, Bronx, New York 10467, USA; \\ ${ }^{9}$ Department of Medicine and Human Oncology and Pathogenesis Program, Memorial Sloan-Kettering Cancer Center, New \\ York, New York 10065, USA; ${ }^{10}$ Department of Surgery, Neurosurgery, and Neurology, Memorial Sloan-Kettering Cancer Center, \\ New York, New York 10065, USA
}

Gene rearrangement in the form of an intragenic deletion is the primary mechanism of oncogenic mutation of the epidermal growth factor receptor (EGFR) gene in gliomas. However, the incidence of platelet-derived growth factor receptor- $\alpha$ (PDGFRA) gene rearrangement in these tumors is unknown. We investigated the PDGFRA locus in PDGFRA-amplified gliomas and identified two rearrangements, including the first case of a gene fusion between kinase insert domain receptor (KDR) (VEGFRII) and the PDGFRA gene, and six cases of PDGFRA ${ }^{\Delta 8,9}$, an intragenic deletion rearrangement. The PDGFRA ${ }^{\Delta 8,9}$ mutant was common, being present in $40 \%$ of the glioblastoma multiformes (GBMs) with PDGFRA amplification. Tumors with these two types of PDGFRA rearrangement displayed histologic features of oligodendroglioma, and the gene products of both rearrangements showed constitutively elevated tyrosine kinase activity and transforming potential that was reversed by PDGFR blockade. These results suggest the possibility that these PDGFRA mutants behave as oncogenes in this subset of gliomas, and that the prevalence of such rearrangements may have been considerably underestimated.

[Keywords: Copy number alteration; glioma; PDGFRA gene rearrangement; receptor tyrosine kinase]

Supplemental material is available at http://www.genesdev.org.

Received July 20, 2010; revised version accepted August 17, 2010.

Although the molecular mechanisms underlying gliomagenesis are rapidly being uncovered, gliomas remain nearly uniformly fatal with the standard of care that includes surgical resection followed by radiation therapy and temozolomide. Many therapeutic trials of signal transduction inhibitors have failed to improve the outcome of gliobastoma multiforme (GBM) patients. This may be the case in part because, although molecular targeted therapy is a rational strategy, GBM comprises a heterogeneous group of tumors, only a subset of which

Corresponding authors.

${ }^{11}$ E-MAIL hollande@mskcc.org; FAX (646) 422-0231.

${ }^{12}$ E-MAIL cbrennan@mskcc.org; FAX (212) 717-3231.

Article is online at http://www.genesdev.org/cgi/doi/10.1101/gad.1972310. responds to any particular inhibitor. Such a possibility makes accurate subdivision of gliomas by signaling characteristics a critical step toward therapeutic success.

Recent analysis of a large number of gliomas has shown that they can be divided into three or four groups by genomic and transcriptomal measures (Phillips et al. 2006; Verhaak et al. 2010), and that these transcriptomal subgroups reflect the common signaling abnormalities found in these tumors (Brennan et al. 2009). Two of the largest subgroups of gliomas are the "classical" GBMs that predominantly harbor epidermal growth factor receptor (EGFR) amplification and rearrangement, and the "proneural" GBMs that are predominantly driven by platelet-derived growth factor (PDGF) signaling. Approximately one-third of the proneural/PDGF tumors show 
amplification of the PDGF receptor $\alpha$ (PDGFRA) locus, while the remainder shows overexpression of the PDGF ligand (Brennan et al. 2009). EGFR and PDGFR inhibition using single agents has limited success in the treatment of unselected GBM patients; however, such an approach may be more effective in particular patients with gliomas harboring genomic activation of either the EGFR or PDGFRA loci (Wen et al. 2006).

Amplification of the EGFR gene is the most frequent genetic alteration of receptor tyrosine kinases (RTKs) found in GBMs $(43 \%)$ and correlates with EGFR overexpression (The Cancer Genome Atlas Research Network 2008). Among GBMs with EGFR overexpression, EGFR gene amplification is identified in $70 \%-90 \%$, many of which additionally express various truncated mutant forms of EGFR, the most common of which is EGFRvIII (Ohgaki and Kleihues 2007). EGFRvIII is generated by an intrachromosomal rearrangement leading to a 267-basepair (bp) in-frame deletion of exons 2-7 in the extracellular domain, leading to ligand-independent constitutive activation (Sugawa et al. 1990; Wong et al. 1992; Ohgaki and Kleihues 2007). This particular mutant form is found in $50 \%-60 \%$ of GBMs with an amplification of the EGFR gene, and is not found in GBMs without amplification or normal tissue (Gan et al. 2009).

In contrast to the common and well-known activating mutations of $E G F R$, reports of activating rearrangements of PDGFRA in GBM are only sporadic. There is one report of an in-frame deletion of the Ig-like domain (PDGFRA ${ }^{\Delta 8,9}$ mutant), and a second with the C-terminal end of PDGFRA (Kumabe et al. 1992; Rand et al. 2005). Additionally, deep sequencing analysis of GBM has also found several point mutations of the Ig-like domain (Verhaak et al. 2010). Although PDGFRA amplification is less common in gliomas than EGFR amplification, PDGFRA gene amplification is found in $11 \%$ of GBMs, making it the second most frequent RTK gene amplified in this family of tumors (The Cancer Genome Atlas Research Network 2008). The question remains as to whether activating rearrangements are as frequent at the PDGFRA locus of PDGFRA-amplified gliomas as they are at the EGFR locus of EGFR-amplified gliomas.

In addition to the intragenic rearrangements described above, the process of gene fusion can also create constitutively active signaling proteins. Historically, most gene fusions were identified in hematological disorders and pediatric sarcomas, and generally were thought to be rare in most solid tumors. However, recent technical advances in array-based analysis have allowed increased cytogenetic characterization of complex rearrangements in solid tumors, as exemplified by the identification of the TMPRSS2-ETS fusion in prostate cancer (Tomlins et al. 2005). Therefore, it is possible that the underrepresentation of gene fusions in solid tumors may be due in part to technical issues rather than pathogenetic differences between them and hematological cancers (Mitelman et al. 2004, 2007; Kumar-Sinha et al. 2006). The only glioma-associated gene fusion reported thus far was a fusion between FIG and ROS identified in the U118MG glioblastoma cell line, and BRAF and
$R A F 1$ gene-associated gene fusions reported recently in childhood pilocytic astrocytomas (Charest et al. 2003; Jones et al. 2008, 2009). To date, a fusion gene has not been identified in adult GBM tumor samples.

In this study, we screened for PDGFRA gene rearrangements in glioma samples, including intragenic deletions and unbalanced fusions. First, based on highresolution array-based comparative genomic hybridization (aCGH) data, we identified several patterns of copy number alterations (CNAs) involving the PDGFRA gene in glioma samples, including the first example of a gene fusion in a human glioblastoma patient sample. This gene fusion, presumably arising from a small paracentric inversion with segmental amplification on chromosome $4 \mathrm{q}$, results in the fusion of the $5^{\prime}$ segment of the kinase insert domain receptor $(K D R)$ gene with the $3^{\prime}$ segment of the PDGFRA gene, generating a KDR-PDGFRA $(K P)$ fusion transcript. We then screened for the PDGFRA ${ }^{\Delta 8,9}$ deletion mutation in gliomas and identified six tumors with this mutation. Of note, all tumors with either the PDGFRA $^{\Delta 8,9}$ or the KP fusion occurred in GBMs with PDGFRA gene amplification. Subsequently, we performed a functional analysis of KP and PDGFRA ${ }^{\Delta 8,9}$ proteins in NIH3T3 fibroblasts and demonstrated that both PDGFRA mutants are transforming oncogenes. The frequency of PDGFRA $^{\Delta 8,9}$ in our series of PDGFRA-amplified GBM was $40 \%$, nearly similar to the frequency of EGFRvIII in EGFRamplified GBM.

\section{Results}

Identification of a novel fusion RTK, KP, in a glioma surgical specimen

While only a subset of gene fusions arising from interchromosomal or intrachromosomal rearrangements are likely to be associated with an alteration in copy number, we hypothesize that these rare events can be identified in a general screen by detecting intragenic transitions in copy number. First, high-resolution aCGH was used to screen for potential rearrangements involving tyrosine kinase (TK) genes in glioma samples. An oligonucleotide microarray was designed to densely cover $89 \mathrm{TK}$ genes for aCGH hybridization. Cases showing intragenic copy number variation between the $5^{\prime}$ and $3^{\prime}$ ends of TK genes were studied further using a second, custom-designed, highdensity CGH array to fine-map the intragenic breakpoints, which eventually guided the identification of the novel gene rearrangements involving these kinases. With the initial screening of 10 glioma tumor samples and 20 cell lines, four gene fusions were identified. These included the known fusion FIG-ROS characterized in the U118MG cell line and serving as a proof of principle for the method (data not shown), and a novel fusion, $K P$, that was isolated from a patient sample and was investigated further in this study.

The aCGH screen identified a complex amplicon within 4q12, spanning the PDGFRA and KDR genes. Both the $P D G F R A$ and $K D R$ genes are localized on chromosome $4 \mathrm{q} 12$ and are transcribed in opposite directions (Fig. 1A,B). The profile suggested amplification of chromosomal 
A

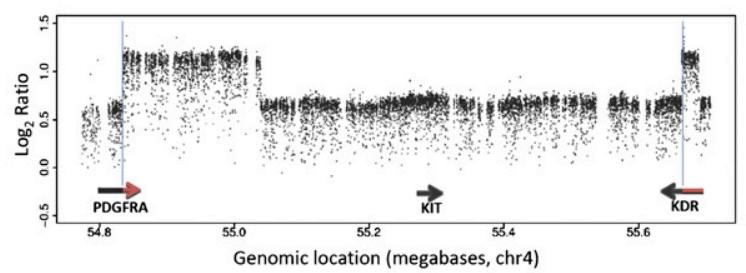

C

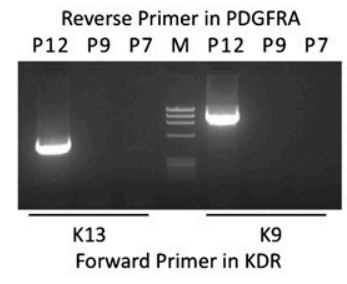

D

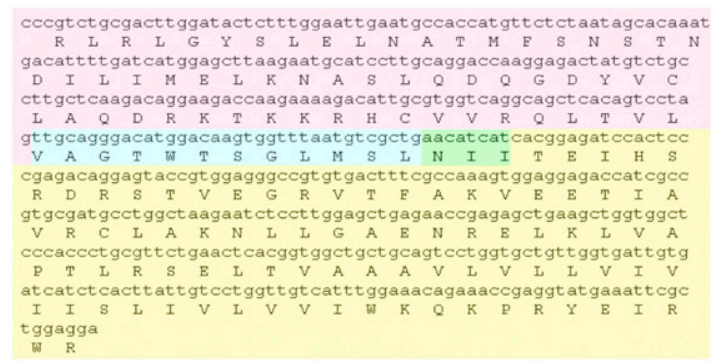

B
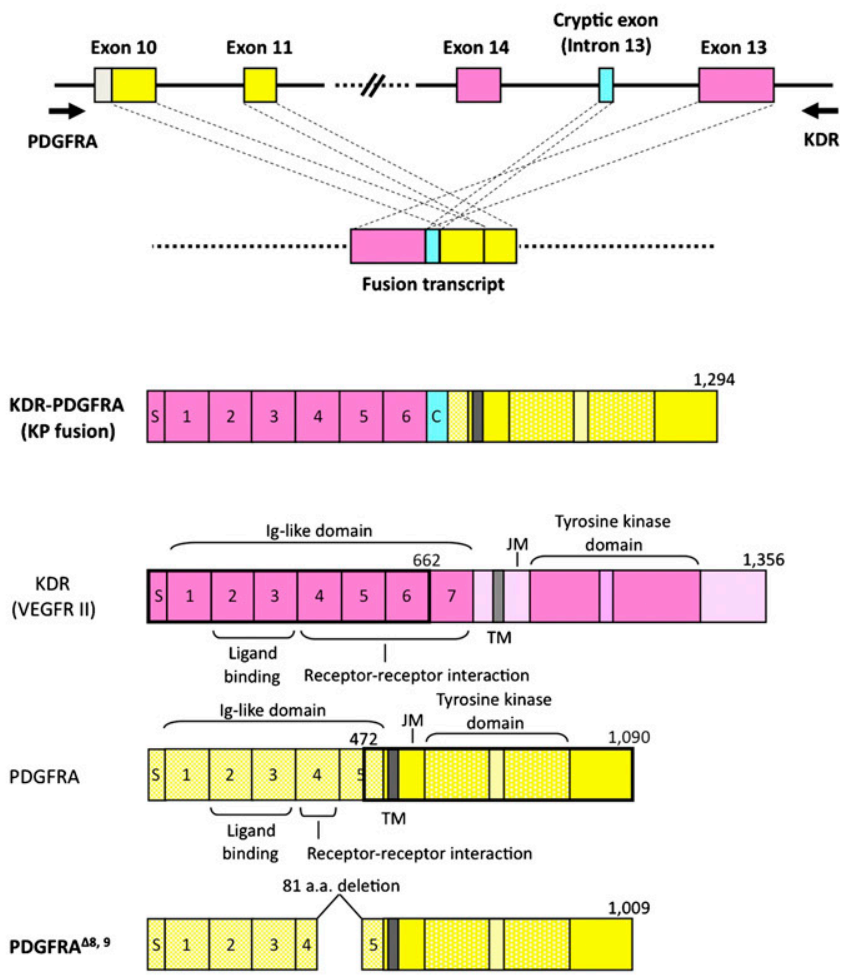

Figure 1. Identification of the novel $K P$ fusion in a glioblastoma tumor. (A) A complex amplicon on chromosome 4 is profiled by highresolution aCGH (median probe interval, $53 \mathrm{bp}$ ). Relative amplification of the $3^{\prime}$ portion of PDGFRA and the $5^{\prime}$ portion of the KDR locus was identified, suggesting possible gene fusion. $(B)$ Schematic diagrams of KDR and PDGFRA partial gene structures as well as the formation of the KP fusion transcript, and of the predicted KP, KDR, PDGFRA, and PDGFRA ${ }^{\Delta 8,9}$ mutant proteins. Because the KDR gene is on the minus strand of chromosome 4, the creation of this fusion appears to have involved an intrachromosomal inversion. (Iglike domain) Immunoglobulin-like domain; (S) signal sequence; (TM) transmembrane domain; (JM) juxtamembrane domain; (C) cryptic exon from $K D R$ intron 13. (C) RT-PCR detection of the KP fusion. The presence of the KP fusion was revealed by RT-PCR using different combinations of KDR forward primers and PDGFRA reverse primers. (M) Molecular marker öX174/HaeIII (Invitrogen); (K9 and K13) forward primers in KDR exons 9 and 13, respectively; (P7, P9, and P12) reverse primers in PDGFRA exons 7, 9, and 12. (D) Partial sequence of the $K P$ fusion transcript. Sequencing of RT-PCR products of K13/P12 and K9/P12 identified the sequence around the fusion point of the KP transcript. The transcript is an in-frame fusion of KDR exon 13 (red) to PDGFRA exon 10 (yellow) with an intervening cryptic exon from $K D R$ intron 13 (blue). The green area indicates 8 bases of overlap sequence between intron 13 of $K D R$ and exon 10 of PDGFRA. The translated amino acid sequence is shown below the transcript sequence.

regions preserving the $5^{\prime}$ end of $K D R$ (excluding the transmembrane and kinase domains) and the kinase domain and $3^{\prime}$ portion of PDGFRA (Fig. 1B). Based on the pattern of copy number changes across this region, we hypothesized that an intrachromosomal rearrangement may have resulted in a gene fusion between KDR and PDGFRA. We reviewed all of the possible exon combinations between $K D R$ and PDGFRA and eliminated those that would produce out-of-frame fusion transcripts. Then, a panel of RTPCR assays was designed to identify the potential fusion transcript in RNA extracted from this tumor. RT-PCR with different gene-specific primers confirmed a fusion transcript in the patient specimen (Fig. 1C). Subsequent sequencing analysis indicated that the breakpoint fell within exon 10 of the PDGFRA gene and intron 13 of the $K D R$ gene, which functions as an in-frame cryptic exon, resulting in an in-frame fusion (Fig. 1B,D). A partial sequence of the KP transcript around the fusion point is shown in Figure 1D, along with the predicted amino acid sequence. Examination of the fusion junction revealed an 8-bp sequence overlap between intron 13 of $K D R$ and exon 10 of PDGFRA (AACATCAT), suggesting microhomologymediated nonhomologous end-joining as a possible mechanism (Fig. 1D; Campbell et al. 2008). Because the KDR gene is on the minus strand of chromosome 4 , the creation of this fusion appears to have involved an intrachromosomal inversion. The PDGFRA copy number transition determined by aCGH aligns with the sequence-based breakpoint within the 60-bp resolution of the platform. The KDR transition is resolved to a 190-bp region of repeat sequence in intron 13 that also harbors the cryptic exon determined by sequencing (Supplemental Fig. S1). The predicted fusion protein consists of an extracellular domain containing the first to sixth Ig-like domain of KDR and the disrupted fifth Ig-like domain of PDGFRA, followed by the intact transmembrane domain and TK 
domain of PDGFRA (Fig. 1B). Subsequently, we screened for the KP fusion by RT-PCR in an additional 205 glioma samples (total, 215 samples), but the KP fusion was not identified in those tumors (data not shown).

\section{PDGFR $A^{\Delta 8,9}$ is a frequent gene rearrangement in PDGFRA-amplified GBM}

$P D G F R A^{\Delta 8,9}$ is formed by an in-frame deletion of $243 \mathrm{bp}$ in exons 8 and 9 of the extracellular portion (Fig. 1B; Kumabe et al. 1992). This type of mutation is reminiscent of the EGFRVIII mutant that is frequently observed in GBM. Given that $P D G F R A^{48,9}$ is a known transforming oncogene due to ligand-independent receptor activation, it is important to determine the frequency of this rearrangement in gliomas (Clarke and Dirks 2003). We initially screened for the PDGFRA ${ }^{\Delta 8,9}$ mutant with RT-PCR analysis in 212 glioma samples from the KP fusion screening. Six cases of PDGFRA ${ }^{\Delta 8,9}$ transcripts were identified in those tumors (Fig. 2A; Supplemental Fig. S2). Interestingly, although five positive tumors expressed both wild-type and deletion mutant (DM) transcripts, one tumor (MSK543) expressed only the DM transcript, possibly suggesting a small homozygous deletion of this locus (Fig. 2A).

In gliomas, amplification of the EGFR gene intimately correlates with not only EGFR overexpression, but also EGFR gene rearrangement (Ekstrand et al. 1991; Mellinghoff et al. 2005). Therefore, we sought to determine the incidence of PDGFRA ${ }^{\Delta 8,9}$ mutation in PDGFRA-amplified gliomas. PDGFRA gene status was determined by quantitative PCR (q-PCR) analysis in the 87 tumors with significant PDGFRA mRNA expression (Supplemental Fig. S2). Gene amplification was identified in 15 out of the 87 cases (Fig. 2B,C). Interestingly, all amplifications were detected in GBMs, and all PDGFRA ${ }^{\Delta 8,9}$ mutants were in these amplified tumors (six out of 15 amplified GBMs; 40\%). Amplification of the PDGFRA gene in these six positive samples was further confirmed with aCGH analysis (data not shown). These results suggest that PDGFRA ${ }^{\Delta 8,9}$ is a recurrent gene rearrangement in PDGFRA-amplified GBM.

\section{Tumors with either PDGFRA gene rearrangement} present characteristic features of oligodendroglioma

Aberrant PDGF signaling induces gliomas with clear oligodendroglioma features in mouse models (Uhrbom et al. 1998; Dai et al. 2001). Therefore, we examined the immunohistochemistry of GBMs with the PDGFRA gene rearrangement to see if robust PDGFRA signaling from the mutant receptor correlates with characteristic morphology of oligodendroglioma, recognized as GBM with oligodendroglial component (GBMO). Tumors with the PDGFRA gene rearrangement (MSK111, KB6, MSK538, and MSK572) demonstrated histological features of oligodendroglioma to various degrees, as supported by the presence of round nuclei, perinuclear halos, minigemistocytes accompanied by prominent PDGFRA, and Olig2 immunopositivity, suggesting PDGF signaling (Fig. 3). As expected, these tumors also showed regions of astrocytic features with diverse level of GFAP expression. In particular, the tumor with the KP fusion expressed higher lev- els of GFAP relative to tumors with the PDGFRA $A^{\Delta 8,9}$ mutation. For comparison, to further investigate whether PDGFRA expression correlates with histological features of oligodendroglioma, we examined the immunohistochemistry of GBMs showing various levels of the PDGFRA transcript. Two samples (MSK261 and KTS2) were found to have the PDGFRA transcript but not PDGFRA gene amplification, and KTS5 had no PDGFRA transcript (Fig. 2A; Supplemental Figs. S2, S3). MSK261 exhibited typical gemistocytic astrocytomas with strong GFAP immunopositivity, while PDGFRA and Olig2 immunopositivity were detected in only subsets of cells. KTS2 had strong GFAP immunopositivity with slight PDGFRA immunopositivity and showed mainly astrocytic features, although few cells with oligodendrocytic features such as perinuclear halos were intermingled. KTS5 had no PDGFRA immunopositivity but a high level of GFAP immunopositivity, showing typical fibrillary astrocytic features. In contrast, MSK352x with PDGFRA amplification included histological features of high-grade oligodendroglioma as well as tumors with the mutant receptor (Fig. 3). Oligodendroglioma (MSK486) showed obvious PDGFRA and Olig2 immunopositivity (Supplemental Fig. S3).

To verify that increased PDGFRA signaling was associated with characteristic morphologies of oligodendroglioma, we compared the histological features of 56 tumors with PDGFRA mRNA expression (Supplemental Table S1). Features of oligodendroglioma were identified in six out of 45 tumors without PDGFRA amplification, and in six out of 11 tumors with PDGFRA amplification (Fisher's exact test, two-sided: $P=0.0075$, comparing the no amplification group with the amplification group), suggesting that excessive PDGFRA signaling correlates with the histological features of oligodendroglioma.

\section{The KP fusion is a constitutively phosphorylated TK} similar to the PDGFRA $A^{\Delta 8,9}$ mutant

Given that the PDGFRA ${ }^{\Delta 8,9}$ protein is autophosphorylated in a ligand-independent manner (Clarke and Dirks 2003), we examined whether the KP fusion behaves as a constitutively active TK as well. We generated retroviral expression vectors encoding the KP fusion and PDGFRA ${ }^{\Delta 8,9}$. These constructs were used individually to infect NIH3T3 mouse fibroblasts, allowing a comparison of the TK activity between the KP fusion and PDGFRA ${ }^{\Delta 8,9}$. In these cells, under serum-deprived conditions, both the KP fusion and PDGFRA $^{\Delta 8,9}$ proteins were autophosphorylated on tyrosine residues and associated with the activation of downstream MAPK and PI3K signaling pathways, as detected by phosphorylated Erk and phosphorylated Akt, respectively (Fig. 4A). In contrast, the activity of these signaling components in cells expressing vector alone, a kinaseinactive mutant of the KP fusion (K831R), wild-type PDGFRA, and wild-type KDR were low (Fig. 4A; Supplemental Fig. S4A).

To further examine whether the KP fusion protein has an intrinsic TK activity, we performed an in vitro kinase assay. As shown in Figure 4B, the KP fusion is capable of autophosphorylation under serum-deprived conditions. 
A

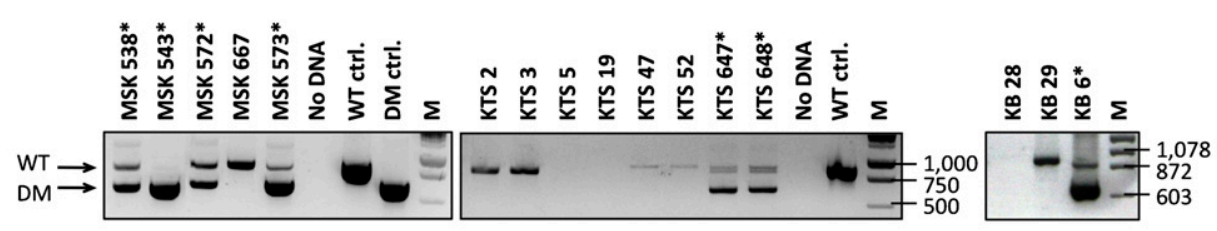

B

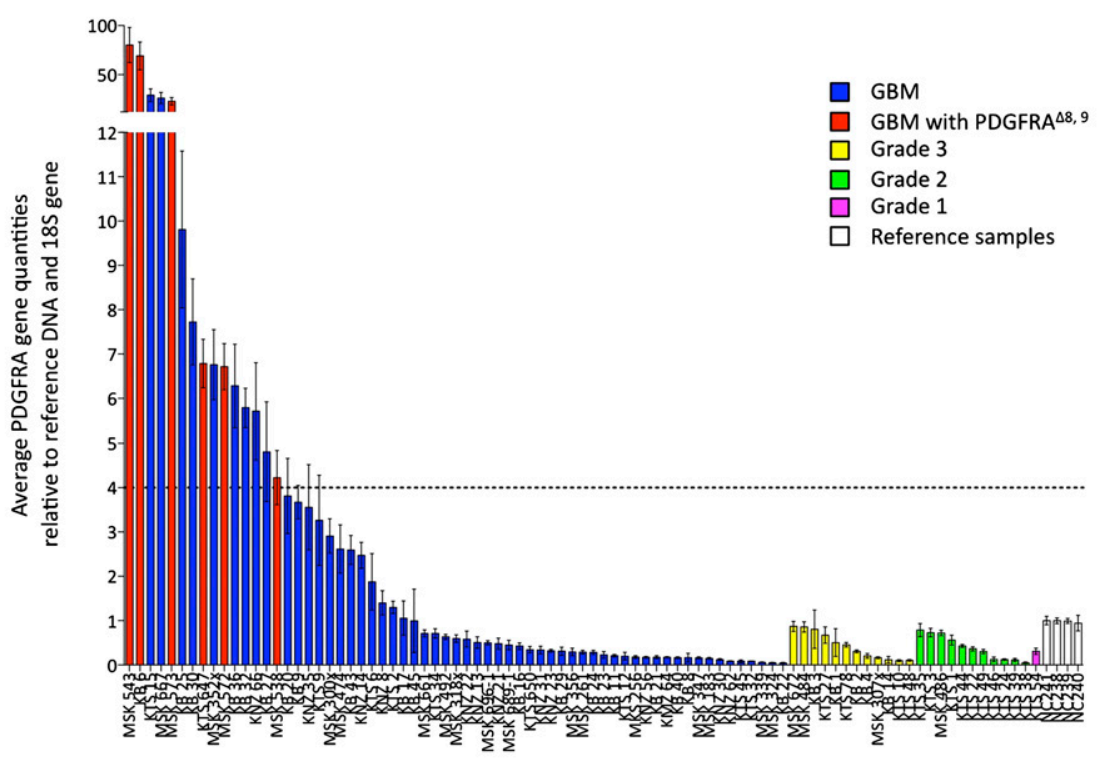

C

\begin{tabular}{|c|c|c|c|c|c|c|c|c|c|}
\hline & No. of Cases & GBM & G3 & $\mathrm{G} 2$ & G1 & $\begin{array}{c}\text { No. of } \\
\text { Amplified TMs } \\
\end{array}$ & $\begin{array}{c}\text { No. of } \\
\text { Amplified GBMs }\end{array}$ & $\begin{array}{c}\text { No. of DM } \\
\text { in Amplified TMs }\end{array}$ & $\begin{array}{c}\text { No. of KP } \\
\text { in Amplified TMs }\end{array}$ \\
\hline KNZ & 13 & 13 & 0 & 0 & 0 & $1 / 13(7.7 \%)$ & $1 / 13(7.7 \%)$ & $0 / 1(0.0 \%)$ & $0 / 1(0.0 \%)$ \\
\hline KB & 25 & 20 & 5 & 0 & 0 & $6 / 25(24.0 \%)$ & $6 / 20(30.0 \%)$ & $1 / 6(16.7 \%)$ & $0 / 6(0.0 \%)$ \\
\hline KTS & 25 & 10 & 4 & 10 & 1 & $2 / 25(8.0 \%)$ & $2 / 10(20.0 \%)$ & $1 / 2(50.0 \%)$ & $0 / 2(0.0 \%)$ \\
\hline MSK & 24 & 20 & 3 & 1 & 0 & $6 / 24(25.0 \%)$ & $6 / 20(30.0 \%)$ & $4 / 6(66.7 \%)$ & $0 / 6(0.0 \%)$ \\
\hline Total & 87 & 63 & 12 & 11 & 1 & $15 / 87(17.2 \%)$ & $15 / 63(23.8 \%)$ & $6 / 15(40.0 \%)$ & $0 / 15(0.0 \%)$ \\
\hline
\end{tabular}

Figure 2. The PDGFRA ${ }^{\Delta 8,9}$ mutant is a recurrent gene rearrangement. (A) RT-PCR detection of the PDGFRA ${ }^{\Delta 8,9}$ mutant in glioma tumors. Identification of the PDGFRA ${ }^{\Delta 8,9}$ mutant was revealed by RT-PCR analysis with a PDGFRA-S3 and PDGFRA-AS2 primer pair that covers an in-frame junction. The putative PCR products of the wild type and the PDGFRA ${ }^{\Delta 8,9}$ mutant are 885 and 642 bp, respectively. Molecular weight (M) is shown in the figure. Sequencing of RT-PCR products from the lower bands of MSK538, MSK543, MSK572, MSK573, KB6, KTS647, and KT648 revealed an in-frame deletion of 243 bp. PMXIG-PDGFRA and PDGFRA ${ }^{\Delta 8,9}$ plasmids were used as controls for wild-type PDGFRA (WT) and the PDGFRA ${ }^{\Delta 8,9}$ mutant (DM), respectively. KTS647 and KTS648 are samples from the same tumor taken from different regions during resection. Samples with the mutation are marked with an asterisk. $(B)$ PDGFRA gene quantitation in GBM tumors. PDGFRA gene amplification was identified using q-PCR analysis in tumors showing high expression of the PDGFRA transcript (Supplemental Fig. S2). The Y-axis indicates average PDGFRA gene quantities relative to the reference sample and $18 S$ gene. Average DNA quantities greater than four were defined as gene amplification (dotted line). (White bars) Normal lymphocytes; (blue bars) GBMs; (red bars) GBMs with the PDGFRA ${ }^{\Delta 8}, 9$ mutant; (yellow bars) grade 3 gliomas, including anaplastic astrocytoma, oligodendroglioma, and ependymoma; (green bars) grade 2 gliomas, including diffuse astrocytoma, oligodendroglioma, mixed oligoastrocytoma, and pilomyxoid astrocytoma; (magenta bars) grade 1 gliomas, including pilocytic astrocytoma. (C) Summary of PDGFRA ${ }^{\Delta 8,9}$ mutant screening. (TMs) Tumors.

In contrast, the tyrosine phosphorylation of the K831R mutant was undetectable under these conditions.

The KP fusion construct used in this experimental setting contains a c-myc or HA epitope in the C-terminal portion of the protein (see below). To exclude the possibility that these epitopes affect the kinase activity of the protein, we analyzed the level of phosphotyrosine using a KP fusion construct without an epitope. Both constructs had similar elevations of phosphotyrosine of the KP fusion, activation of the MAPK and PI3K signaling pathways, and transformed cell morphology (see below; data not shown).

The KP fusion has an intact kinase domain of PDGFRA. Therefore, to investigate whether the KP fusion behaves as a constitutively active PDGFRA, we compared the KP fusion-induced activation of the MAPK and PI3K pathways under serum-deprived conditions with WT-PDGFRA and WT-KDR after cognate ligand stimulation. As shown 


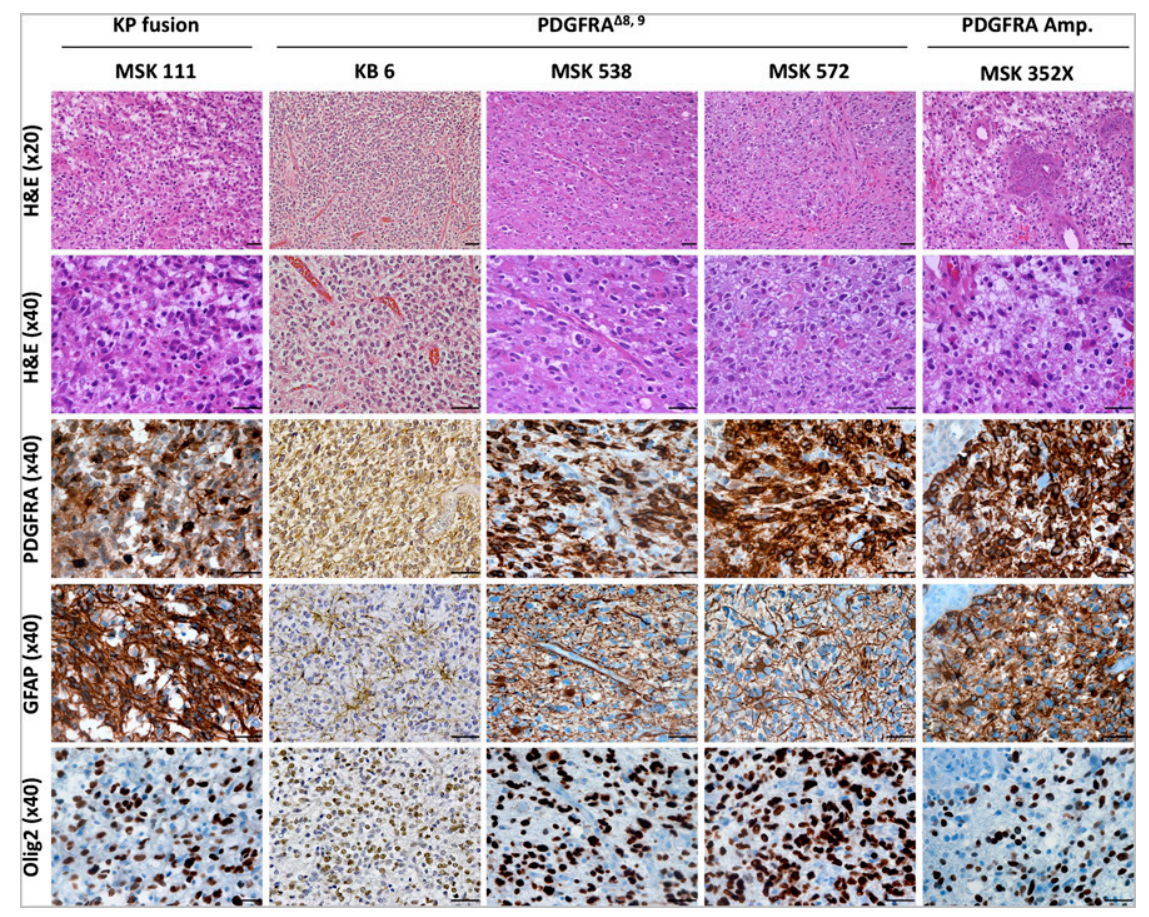

Figure 3. Histopathological features of glioblastomas with the PDGFRA mutant. Representative images of H\&E, PDGFRA, GFAP, and Olig2 staining are shown at $20 \times$ or $40 \times$ magnification. Tumors with the PDGFRA mutation demonstrate histological features of oligodendroglioma, as shown by round nuclei, perinuclear halos, a chicken wire-like capillary network, and minigemistocytes with prominent Olig2 expression. GBM (MSK352x) has a PDGFRA transcript with PDGFRA amplification. Bars, $50 \mu \mathrm{m}$. in Figure 4, A and C, and Supplemental Figure S4A, the cells expressing the KP fusion (Fig. 4C, lane 3) exhibited ligand-independent elevated activation of the MAPK and PI3K pathways compared with either the mock controls (Fig. 4C, lane 1) or cells expressing the K831R mutant (Fig. 4C, lane 2). PDGF ligand stimulation of WT-PDGFRA activated both the MAPK and PI3K pathways, similar to $\mathrm{KP}$ fusion-expressing cells (Fig. 4C, lanes 3,4). In contrast, WT-KDR expression alone had no effect, and VEGF ligand stimulation of WT-KDR activated mainly the MAPK pathway in these cells (Fig. 4C, lane 5; Supplemental Fig. S5D).

Many kinases have some basal level of activity. NIH3T3 cells express endogenous PDGFRs but not KDR, leaving open the formal possibility that wild-type PDGFRs affect the kinase activity of the KP fusion (Supplemental Fig. S4B). Therefore, we investigated the level of phosphotyrosine and principal downstream signaling pathways of the KP fusion in Cos 7 cells that express a very low level of endogenous PDGFRB but not PDGFRA or KDR (Supplemental Fig. S4B). In Cos 7 cells under serum-deprived conditions, the KP fusion showed elevated tyrosine phosphorylation and PI3K pathway activation (Fig. 4D, left panel). In addition, to determine if the KP fusion functions similarly in glioma cells, we tested this construct in the T98G glioma cell line (Fig. 4D, middle panel) and primary glial cells (Fig. 4D, right panel). In both cell types, under serum-deprived conditions, forced expression of either the KP fusion or PDGFRA ${ }^{\Delta 8,9}$ produced elevated tyrosine phosphorylation of both mutants and PI3K pathway activation. Collectively, these results suggested that the KP fusion behaves as a constitutively active TK similar to the PDGFRA $^{\Delta 8,9}$ mutant.
The KP fusion can form homodimers and heterodimers in a ligand-independent manner

Ligand binding to its cognate receptor leads to receptor dimerization and tyrosine autophosphorylation, followed by its activation. These phosphorylated tyrosine residues mediate the specific binding of cytoplasmic signaling proteins containing Src homology-2 (SH2) and protein tyrosine-binding (PTB) domains (Blume-Jensen and Hunter 2001). The above data indicate that the KP fusion behaves as a constitutively active TK in a ligand-independent manner, implying that the KP fusion might constitutively exist in a dimeric form.

To test this hypothesis, we performed immunoprecipitation analysis with NIH3T3 and 293 cells coexpressing HA-tagged KP fusion and c-myc-tagged KP fusion vector constructs under serum-deprived conditions, and then probed immunoblots with the indicated antibodies (as shown in Fig. 4E,F). The homodimer of the KP fusion was readily observed in both NIH3T3 and 293 cells coexpressing the c-myc-tagged KP fusion construct together with the HA-tagged KP fusion (Fig. 4E,F, both panels, lane 2).

PDGFR can form a heterodimeric receptor complex between the $\alpha$ and $\beta$ receptors (Rupp et al. 1994). In addition, PDGFRA and PDGFRB can also form heterodimers with other RTK family members, as demonstrated for FGF-R1 and EGFR, respectively (Saito et al. 2001; Faraone et al. 2006). Therefore, we used immunoprecipitation/Western blot analysis to address the possibility that the KP fusion could form heterodimers with PDGFRA or KDR under serum-deprived conditions. The KP fusion formed heterodimers together with tyrosine phosphorylated forms of either PDGFRA or KDR (Fig. 4F, both panels, lanes 3,4$)$. These results suggest that the KP 
A

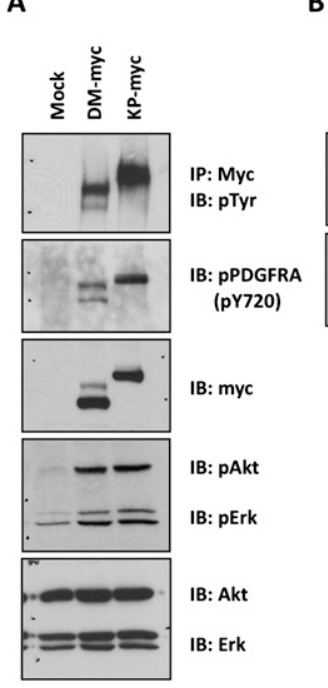

C

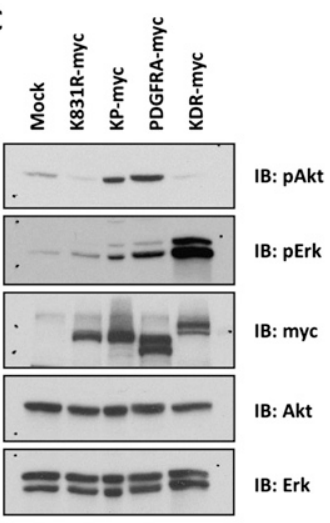

B

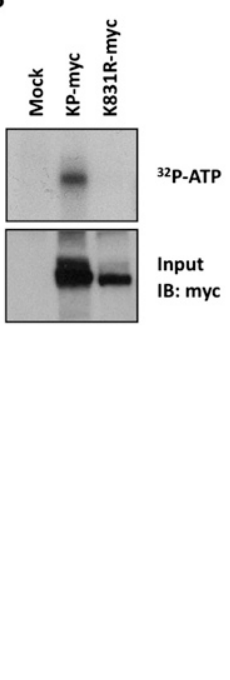

D

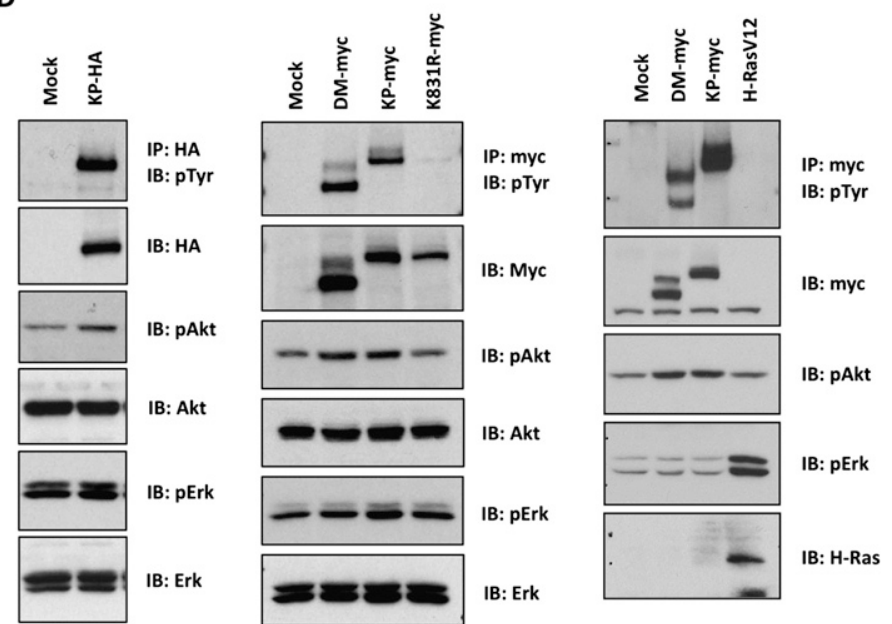

E
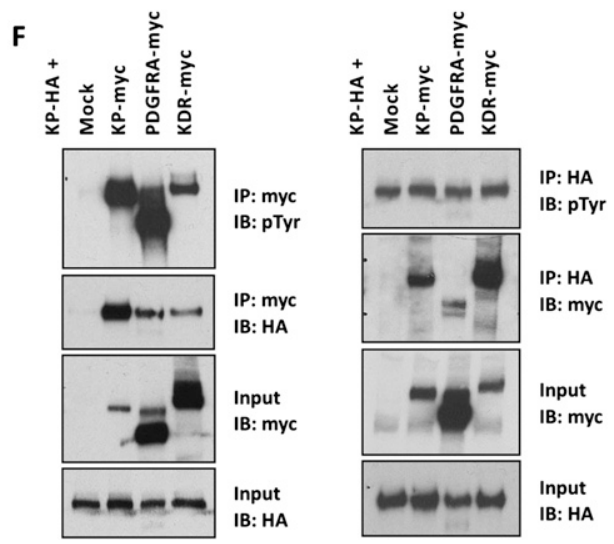

Figure 4. (A) The KP fusion (KP) and PDGFRA ${ }^{\Delta 8,9}$ mutant (DM) are both constitutively active TKs. (Top panel) After $24 \mathrm{~h}$ of serumfree conditions, cells expressing mock (lane 1), the PDGFRA ${ }^{\Delta 8,9}$ mutant (lane 2), and the KP fusion (lane 3) were immunoprecipitated (IP) with anti-c-myc agarose, and the immunoblots (IB) were probed with anti-phosphotyrosine antibody. Whole-cell lysates were assayed with the indicated antibodies for input of immunoprecipitation or detection of phosphotyrosine (Y720) and downstream signaling. (B) In vitro kinase assay of NIH3T3 cells expressing mock (lane 1), the KP fusion (lane 2), and the kinase-inactive mutant (K831R) of the KP fusion (lane 3). (Top panel) Whole-cell lysates from each cell line were immunoprecipitated with anti-c-myc agarose after $24 \mathrm{~h}$ of serum-free conditions, and then subjected to an in vitro kinase reaction with $\left[\gamma^{-}{ }^{32} \mathrm{P}\right]$ ATP. (Bottom panel) Whole-cell lysates were also subjected to immunoblot analysis with anti-c-myc antibody for input of immunoprecipitation. Autophosphorylation of the KP fusion was detected by autoradiography. $(C)$ The KP fusion behaves as a constitutively active PDGFRA. Cells expressing mock (lane 1), the KP fusion (lane 2), and the kinase-inactive mutant (K831R) of the KP fusion (lane 3) were harvested after $24 \mathrm{~h}$ of serum-free conditions. As a control, the cells expressing WT-PDGFRA (lane 4) and WT-KDR (lane 5) were stimulated with PDGF-BB (PB) or VEGF165 for $5 \mathrm{~min}$ after $24 \mathrm{~h}$ of serum-free conditions, respectively. The cells were then subjected to immunoblot analysis with the indicated antibodies. $(D)$ The KP fusion behaves as an active kinase in various cells. (Left panel) Cos 7 cells. (Middle panel) T98G glioma cells. (Right panel) primary glial cells. DNA plasmids indicated were introduced with transient transfection (Cos 7 cells) or with retroviral infection (T98G and primary glial cells). Cos 7 cells were incubated for $12 \mathrm{~h}$ in serum-free DMEM after $60 \mathrm{~h}$ of transfection, and were immunoprecipitated with anti-HA agarose. Whole-cell lysates were then subjected to immunoblot analysis with the indicated antibodies. T98G and primary glial cells were immunoprecipitated with anti-myc agarose after 12 or $24 \mathrm{~h}$ of serum-free conditions, and were then subjected to immunoblot analysis with the indicated antibodies as well. Cells expressing H-RasV12 were used as a control of the experiment. $(E)$ Immunoprecipitation analysis of KP fusion homodimerization in NIH3T3 cells. (F) Immunoprecipitation analysis of KP fusion homodimerization and heterodimerization in 293 cells. Representative blots from reproduced experiments are shown.

fusion can allow receptor homodimerization and heterodimerization under conditions of overexpression, possibly leading to the activation of not only the KP fusion itself but also PDGFRA and KDR.

The PDGFRA ${ }^{\Delta 8,9}$ DM shows a predominantly cytoplasmic localization (Clarke and Dirks 2003). Therefore, we examined whether the KP fusion, as either a ligand-independent homodimer or a heterodimer with PDGFRA and KDR, is also predominantly localized to the cytoplasm. Subcellular localization was determined by immunocytochemistry following transient cotransfection of Cos 7 cells using the HA-tagged KP fusion construct with various c-myctagged receptor constructs under serum-deprived conditions. By itself, the KP fusion localized mainly to vesicles 
in the cytoplasm, especially concentrated around the perinuclear region (Supplemental Fig. S4C, panels b,d-f). Both the HA-tagged KP fusion and c-myc-tagged KP fusion colocalized completely (Supplemental Fig. S4C, panels d-f). Immunofluorescence analysis in serum-deprived conditions for WT-PDGFRA alone or WT-KDR alone revealed their cell surface localization in some cells along with uniform or punctate staining distributed in the cytoplasm and/or perinuclear region (Supplemental Fig. S4C, panels n,o). When either WT-PDGFRA or WTKDR was coexpressed with the KP fusion, the amount of wild-type receptors present on the cell surface was somewhat reduced and resulted in partial colocalization with the KP fusion in the cytoplasm (Supplemental Fig. S4C, panels $\mathrm{j}-1$ ). These results suggest that the KP fusion protein may to some degree trap PDGFRA and KDR in the cytoplasm, depending on expression levels, and may contribute to activation of these receptors in that subcellular location.

The KP fusion protein has ligand-independent transforming potential

Given that PDGFRA ${ }^{\Delta 8,9}$ is a transforming oncogene (Clarke and Dirks 2003), we determined whether the KP fusion may also have transforming potential. We found that NIH3T3 cells expressing the KP fusion were morphologically transformed, as demonstrated by spindle morphology, a loss of contact inhibition, and formation of foci in a pattern similar to the cells expressing the PDGFRA $^{\Delta 8,9}$ mutant (Figs. 5A [panels b,c,f,g], 6B,D). In contrast, the morphology of cells expressing the kinasedead (K831R) mutant of KP was comparable with cells expressing vector alone (Fig. 5A, panels a,d,e,h). Interestingly, the transformed phenotype of the KP fusion increased with prolonged passage in culture, eventually forming distinct foci very similar to typical Ras-transformed cells. In this process, MAPK signaling inversely decreased with prolonged passage, and PI3K signaling became dominant under serum-deprived conditions (data not shown). Therefore, we performed a series of experiments with the cells of a lower passage number to obtain reproducible results in this study. We grew these cells in soft agar to examine whether cells expressing the KP fusion could proliferate in anchorage-independent conditions. The cells expressing the KP fusion maintained growth and formed colonies in soft agar similar to those expressing PDGFRA $^{\Delta 8,9}$ (Fig. 5B, panels b,c,f,g). In contrast, the cells expressing mock or the K831R mutant could not grow in soft agar with $5 \%$ serum conditions (Fig. $5 \mathrm{~B}$, panels $\mathrm{a}, \mathrm{d}, \mathrm{e}, \mathrm{h})$.

To investigate the tumorigenic potential of the KP fusion in an allograft model in vivo, we implanted NIH3T3 cells expressing the KP fusion into the flank of nude mice. When $10^{5}$ cells were inoculated, tumors developed as a small nodule within $\sim 2$ wk in all mice (Fig. 5C). NIH3T3 cells $\left(10^{5}\right)$ expressing vector alone or the K831R mutant of the KP fusion formed small nodules in our experimental model, and grew statistically slower and smaller than NIH3T3 cells expressing the KP fusion (Fig. 5C,D). These results further support the notion that the KP fusion functions as a potent oncogene similar to PDGFRA $^{\Delta 8,9}$, and that the kinase activity is necessary for oncogenic transformation in NIH3T3 cells.

Both PDGFRA mutants functioned as active kinases in primary Ink4/Arf ${ }^{-/}$glial cells as well as NIH3T3 cells

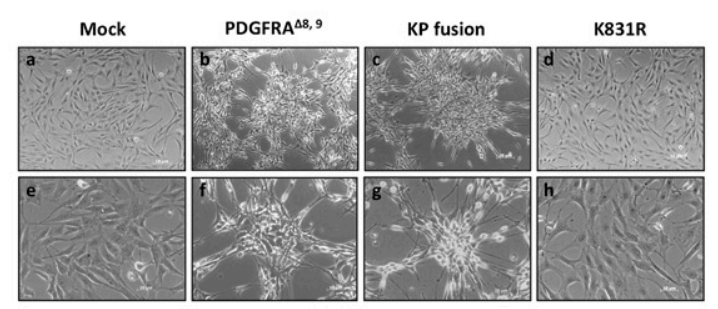

B

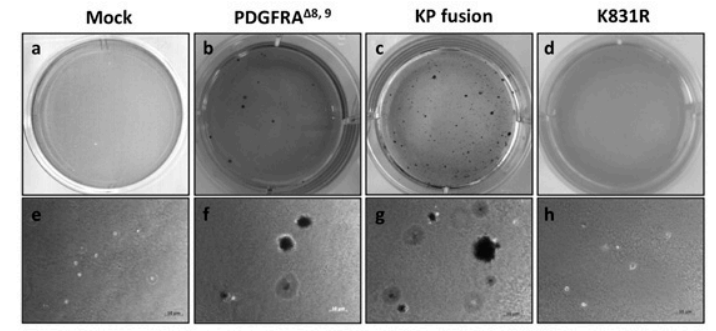

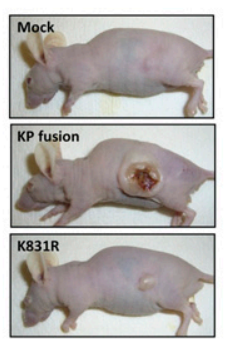

D

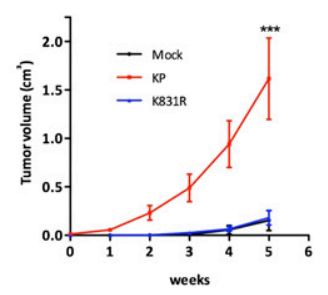

Figure 5. The KP fusion gene is a transforming oncogene. $(A)$ Cells expressing the KP fusion are morphologically transformed. Cell morphology of NIH3T3 cells expressing mock (panels $a, e$ ), the PDGFRA ${ }^{\Delta 8,} 9$ mutant (panels $b, f$ ), the KP fusion (panels $c, g)$, and the kinase-inactive mutant (K831R) of the KP fusion (panels $d, h$ ) were photographed at $100 \times($ top panels) or $200 \times$ (bottom panels) magnification (bars, $10 \mu \mathrm{m}) .(B)$ Analysis of anchorage-independent growth. NIH3T3 cell lines described in $A$ were maintained on soft agar with $5 \%$ calf serum; colony formation in soft agar is shown with $1 \times($ top, panels $a-d)$ or $100 \times$ (bottom, panels $e-h$ ) magnification after 4 wk (bars, $10 \mu \mathrm{m}) .(C)$ Tumor formation in nude mice. Cell lines as described in $A$ were inoculated subcutaneously into the flanks of nude mice. Each tumor was measured weekly and was photographed after 6 wk of inoculation. $(D)$ The growth curve of tumors as described in $C$. The $\mathrm{KP}$ fusion $(n=6)$ was compared with mock $(n=6)$ or K831R $(n=$ 4) at the final point, respectively. $\left(^{\star \star \star}\right) P<0.001$. Tumor formation of cells expressing the KP fusion was reproduced four times in an independent experiment. Representative results are shown in $C$ and $D$. 
(Fig. 4D, right panel). However, these primary glial cells neither displayed a transformed phenotype nor formed colonies in soft agar upon expression of either the KP fusion or PDGFRA ${ }^{\Delta 8,9}$ (data not shown). These results suggested that expression of these PDGFRA mutants alone is insufficient for transformation of glial cells, consistent with their occurrence being limited to high-grade tumors.

\section{The transforming activity of the PDGFRA mutants is reversed by the PDGFR kinase inhibitors Gleevec and PTK787}

Consistent with the observation that the KP fusion localizes mainly to the cytoplasm (Supplemental Fig. S4C), a neutralizing antibody to the extracellular domain of KDR did not affect tyrosine phosphorylation and colony formation in soft agar of NIH3T3 cells expressing the KP fusion (Supplemental Fig. S5B,C), while this same antibody treatment blocked phosphorylation of WT-KDR (Supplemental Fig. S5A). VEGF stimulation showed a slight increase in the tyrosine phosphorylation of the KP fusion; possibly a small percent of the KP fusion protein is capable of reaching the cytoplasmic membrane and binding with the ligand for activation of the receptor (Supplemental Fig. S5D). On the surface, the data suggest that the KP fusion acts in a ligand-independent fashion. However, it is possible that the KP fusion interacts with RTKs that require binding of their respective ligands for activation.

The KP fusion has an intact protein kinase domain of PDGFRA and may require TK activity of PDGFRA for its transforming potential, as shown in Figure 5. Therefore, we addressed the effect of two RTK inhibitors with known activity against PDGFR: Gleevec and PTK787 (Druker et al. 1996; Wood et al. 2000). Both PTK787 (Fig. 6A; Supplemental Fig. S5E, left panel) and Gleevec (Fig. 6A; Supplemental Fig. S5E, right panel) effectively reduced the level of phosphotyrosine together with active downstream signaling in NIH3T3 cells expressing the KP fusion in a concentration-dependent manner. Furthermore, $1 \mu \mathrm{M}$ PTK787 treatment partially reverted the KP fusioninduced transformed phenotype to the untransformed morphology, and $10 \mu \mathrm{M}$ treatment reverted this phenotype completely (Fig. 6B). PTK787 also inhibited anchorage-independent growth of NIH3T3 cells expressing the $\mathrm{KP}$ fusion beginning at $1 \mu \mathrm{M}$ concentration and completely inhibited them at $10 \mu \mathrm{M}$ (Fig. 6C), while $1 \mu \mathrm{M}$ Gleevec completely inhibited the KP fusion-induced transformed phenotype (Fig. 6B,C). In addition, we addressed the inhibitory effect of both PDGFR inhibitors on the PDGFRA $^{\Delta 8,9}$ mutant, which has an intact protein kinase domain of PDGFRA. Both Gleevec and PTK787 showed an inhibitory effect on the elevated tyrosine phosphorylation of the mutant itself and the active downstream signals (Fig. 6A; Supplemental Fig. S6E), and on the transformed phenotype of the PDGFRA ${ }^{\Delta 8,9}$ mutant, similar to cells expressing the KP fusion (Fig. 6B,C). These results further support the idea that both the KP fusion and PDGFRA ${ }^{\Delta 8,9}$ mutants behave as constitutively active PDGFRAs, and that the transformed phenotype is dependent on the TK activity of both mutant receptors.
The activity of both the MAPK and PI3K signaling pathways is essential for the transforming potential of both PDGFRA mutants

Both Gleevec and PTK787 inhibited the elevated tyrosine phosphorylation of the PDGFRA mutants and the mutant-induced downstream signal transduction activity (Fig. 6A; Supplemental Fig. S5E). Therefore, to examine whether either MAPK or PI3K activity is required for the transforming potential of PDGFRA mutants, we determined the effect of the MEK inhibitor U0126 and the PI3K inhibitor LY294002 on the morphology of NIH3T3 cells expressing the PDGFA mutants, respectively (Fig. 6D). Initially, to confirm the inhibitory effects of individual inhibitors on the PDGFRA mutant-induced downstream signaling pathways, we performed immunoblot analysis on cells after treatment with the individual inhibitors (Supplemental Fig. S5F). After $72 \mathrm{~h}$ of treatment, PI3K inhibition alone could not completely reverse the transformed phenotype of either of the PDGFRA mutant cells, as shown by cluster formation and loss of contact inhibition. The MEK inhibitor alone also gave an incomplete reversal of the transformed phenotype, as shown by the partial relief of cluster formation, although it was more effective than PI3K inhibition alone. In contrast, combined inhibition of both the MAPK and PI3K pathways resulted in complete phenotypical reversion of cells expressing the PDGFRA mutants, yielding the same flat morphology as the NIH3T3 cells expressing mock vector or PDGFRA mutant expressors treated with PDGFR inhibitors (Figs. 5A, 6B,D). To further dissect the downstream signaling of the PI3K pathway, we used the mTOR inhibitor rapamycin. Neither rapamycin treatment alone nor its combination with LY294002 completely reversed the morphology of NIH3T3 cells expressing the PDGFRA mutants. In contrast, when rapamycin was used together with U0126, the cluster formation of PDGFA mutants was partly decreased, but the spindle-shaped morphology was still maintained, possibly through signaling from the PI3KAkt pathway rather than via mTOR (Fig. 6D). These results suggested that the transforming potential of PDGFRA mutants might be mediated by a combination of both MAPK and PI3K pathway activation originating from enhanced PDGFRA signaling.

\section{Discussion}

Although the common internal deletion of the EGFR gene, EGFRVIII, has been frequently identified and shown to contribute to glioma progression, the PDGFRA gene rearrangement has been thought, to date, to be a rare event. The functionality between aberrant PDGFRA signaling and gliomagenesis remains to be clarified despite the fact that it is the second most frequently amplified gene in GBMs (Sugawa et al. 1990; Wong et al. 1992; The Cancer Genome Atlas Research Network 2008). In the present study, we investigated two activating PDGFRA gene rearrangements in gliomas. First, we identified the first gene fusion in GBM surgical specimens, between KDR and PDGFRA, by aCGH, detecting them as intragenic copy 
A

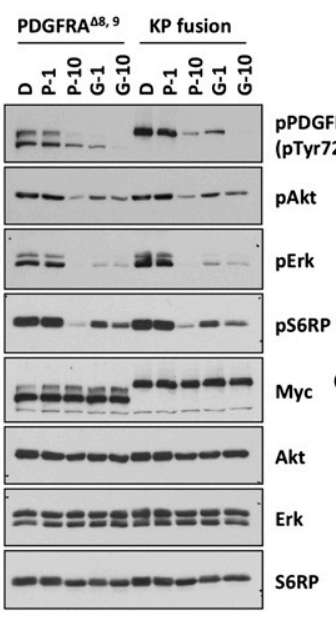

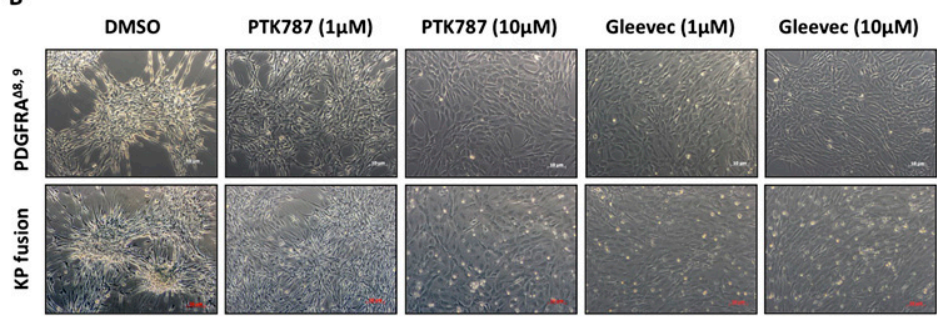

D

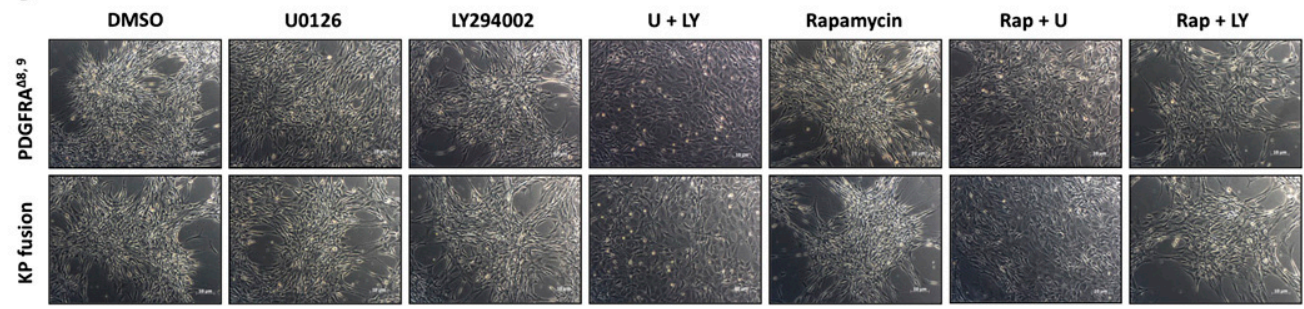

Figure 6. PDGFRA mutants are potential therapeutic targets of the RTK inhibitors PTK787 and Gleevec. (A) PTK787 and Gleevec inhibit tyrosine phosphorylation with active downstream signals of PDGFRA mutants in a concentration-dependent manner. Cells expressing the PDGFRA ${ }^{\Delta 8,9}$ mutant or KP fusion were treated with 0.1\% DMSO (D) and PTK787 (P1: $1 \mu$ M PTK787; P10: $10 \mu M$ PTK787) or Gleevec (G1: $1 \mu \mathrm{M}$ Gleevec; G10: $10 \mu \mathrm{M}$ Gleevec) at the indicated drug concentrations. After $2 \mathrm{~h}$ of treatment, the cells were harvested and whole-cell lysates were subjected to immunoblot analysis with the indicated antibodies. A representative blot is shown. (B) The effects of PTK787 and Gleevec on the transformed phenotype of NIH3T3 cells expressing PDGFRA mutants. Cells expressing the KP fusion or PDGFRA ${ }^{\Delta 8,9}$ mutant were treated with $0.1 \%$ DMSO, PTK 787 , or Gleevec at the indicated drug concentrations. Cell morphology was observed and documented at $100 \times$ magnification after $72 \mathrm{~h}$ of individual treatment $(\mathrm{bars}$, $10 \mu \mathrm{m}) .(C)$ The effects of PTK787 and Gleevec on anchorage-independent growth of PDGFRA mutants. Cells expressing the KP fusion or PDGFRA ${ }^{\Delta 8,9}$ mutant were maintained on soft agar with $5 \%$ calf serum containing $0.1 \%$ DMSO, PTK787, or Gleevec at the indicated drug concentrations. Fresh medium containing $5 \%$ calf serum with DMSO, PTK787, or Gleevec was added onto the upper agar every $4 \mathrm{~d}$. The colonies were photographed at $100 \times$ magnification after $3 \mathrm{wk}$. DMSO control was compared with $1 \mu M$ PTK787 treatment against the KP fusion or PDGFRA ${ }^{\Delta 8,9}$ mutant cells, respectively. $\left.\left({ }^{\star \star}{ }^{\star}\right) P<0.0001\right)$. Bars, $10 \mu \mathrm{m}$. (D) The effect of treatment with various inhibitors on the transformed phenotype induced by PDGFRA mutants. The NIH3T3 cells expressing the KP fusion or PDGFRA ${ }^{\Delta 8,9}$ mutant were treated with $0.1 \%$ DMSO, $10 \mu \mathrm{M}$ U0126, $10 \mu \mathrm{M}$ LY294002, 1 nM rapamycin, and the indicated combinations of inhibitors. Cell morphology was photographed at $100 \times$ magnification after $72 \mathrm{~h}$ of individual treatment (bars, $10 \mu \mathrm{m})$.

number changes in an individual gene. In addition, we demonstrated that the previously identified PDGFRA ${ }^{\Delta 8,9}$ is a recurrent gene rearrangement occurring in $40 \%$ of GBMs with amplified PDGFRA, a rate nearly similar to that found for EGFRVIII in the subset of EGFR-amplified gliomas. Other PDGFRA gene rearrangements may exist in GBMs that have not yet been identified.

PDGF signaling contributes to tumor formation in a subset of GBMs, some of which are driven by the PDGF ligand, while others are driven by genetic alterations of the PDGFRA gene, such as gene amplification, intrachromosomal deletion, and activating point mutation. Our data indicate that the KP fusion and PDGFRA ${ }^{\Delta 8,9}$ represent two examples of these genetic mechanisms in this subset of PDGFRA-driven GBMs.
Several observations suggest that these PDGFRA mutations can contribute to the formation or progression of these particular tumors: First, these rearrangements appear to have been selected for in these tumors; second, both the KP fusion and the PDGFRA ${ }^{\Delta 8,9}$ mutant are experimentally oncogenic; and, finally, dysregulated PDGF signaling is causal for glioma formation in mice (Uhrbom et al. 1998; Dai et al. 2001). We examined the effect of PDGFR inhibitors on cells expressing PDGFRA mutants. These inhibitors effectively reverse the mutant receptor-mediated cellular transformation of these cells. Although PDGFR inhibition alone has not been successful in the treatment of unselected GBM patients, such an approach may have had beneficial effects in cases of patients having gliomas with similar rearrangements at this locus. However, such 
optimism should be tempered by the feeble response of EGFR mutant GBM patients to EGFR inhibition, as well as the capacity for other coactivating RTKs to swap in upon extinction of any one RTK (Stommel et al. 2007).

Expression of constitutively active versions of EGFR by themselves does not form gliomas in experimental mouse models; these mutations must be combined with additional genetic lesions, such as Ink4/Arf deletion, for EGFR-induced glioma formation (Holland et al. 1998; Zhu et al. 2009). Moreover, all GBMs with EGFR gene rearrangement have amplified wild-type $E G F R$ genes as well, suggesting that the wild-type allele of EGFR may contribute to the oncogenic effect of the mutant allele (Mellinghoff et al. 2005). The same pattern is seen with rearrangements in PDGFRA, most of which were associated with the amplification of the wild-type PDGFRA gene as well. It is possible that amplification and elevated expression of wild-type receptors may be essential for the oncogenic potential of such mutant receptors.

Dysregulated PDGF ligand expression induces oligodendroglioma-like gliomas in mouse models (Uhrbom et al. 1998; Dai et al. 2001). However, the glial cells expressing either the KP fusion or PDGFRA ${ }^{\Delta 8,9}$ did not transform even if they had an Ink4/Arf ${ }^{-/-}$genetic background, for unknown reasons (data not shown). This discrepancy is consistent with the fact that, although the PDGF ligand is overexpressed in gliomas of all grades, these receptor mutations are only late in the progression of the tumors in humans. In mouse models, the PDGF ligand is fundamentally different from a constitutively active PDGFRA because the PDGF ligand is capable of activating not only glia by autocrine stimulation, but also other cell types, such as endothelia, by paracrine stimulation. The discrepancy between the oncogenic effect on NIH3T3 cells and glial cells suggests that the sufficiency of these PDGFRA mutants as transforming agents is cell type-specific.

Histologic analysis of the tumors expressing PDGFRA mutants showed characteristic features of oligodendroglioma to various degrees, possibly corresponding to the GBM subtype "GBM with oligodendroglioma component" in the World Health Organization (WHO) classification. Genomic analysis of this subtype revealed that there were no distinguishable genetic alterations from GBMs without an oligodendroglioma component (Homma et al. 2006). Our present study suggests that abnormal PDGFRA signaling is likely to contribute to histological features of oligodendroglioma, a notion supported by the induction of similar gliomas by forced PDGFB ligand in mouse models (Uhrbom et al. 1998; Dai et al. 2001). However, from a therapeutic standpoint, the histological characteristics of oligodendroglioma with PDGFRA gene rearrangement may be useful as a marker in the selection of PDGFR inhibitor treatment in GBMs.

The KP fusion formed a heterodimer with wild-type PDGFR or wild-type KDR and, to various degrees, colocalized with them in the cytoplasm, possibly contributing to enhance wild-type receptor activity in that location. It is known that RTKs can form heterodimers with other RTK family members at the cell surface, and such heterodimers can activate signaling pathways (Saito et al. 2001; Faraone et al. 2006). In particular, PDGFR and VEGFR belong to the type III RTK family (the immunoglobulin superfamily) characterized by Ig-like domains in their extracellular region necessary for receptor-receptor interaction. Therefore, heterodimer formation of PDGFR or KDR with the KP fusion might be similar to the heterodimer formed between PDGFRB and FGFR1, which also belong to the immunoglobulin superfamily (Faraone et al. 2006). In addition, given that the seventh Ig-like domain of KDR is required for inhibition of receptor dimerization (Tao et al. 2001), the deletion of this sequence in the KP fusion also may relieve the inhibition, resulting in increased dimerization between the KP fusion and itself or other RTKs.

The KP fusion localized predominantly in the cytoplasm, as indicated by the lack of effect of anti-KDR antibody treatment of these cells. The PDGFRA ${ }^{\Delta 8,9}$ mutant also shows a predominantly cytoplasmic localization (Clarke and Dirks 2003). This mutant has an in-frame deletion in the extracellular region, resulting in loss of approximately half of the fourth Ig-like domain and half of the fifth Ig-like domain. Thus, the defect of the fourth or fifth Ig-like domain of PDGFRA as well as the KP fusion may affect its subcellular localization differently than the plasma membrane-localized EGFRvIII mutant or other RTK family members that do not have Ig-like domains. A majority of the KP fusion appears not to reach the cell surface and sequesters the wild-type receptor in the cytoplasm, as shown by heterodimerization with predominantly phosphorylated forms of the wild-type receptor.

EGFRvIII functions as an activated monomer and uses an altered spectrum of signaling cascades for oncogenic transformation relative to the wild-type EGFR dimer (Chu et al. 1997). In contrast, the KP fusion exists in a dimeric form and shows the same activation pattern of downstream signaling as wild-type PDGFRA, implying the possibility that heterodimer formation between the KP fusion and endogenous PDGFR might enhance PDGFR signaling and drive cellular transformation of NIH3T3 cells. It is possible that wild-type PDGFR may be needed for the transformed phenotype of NIH3T3 cells that normally express endogenous PDGFRs.

Gliomas are a molecularly heterogeneous disease. The identification and analysis of these PDGFRA rearrangements illustrates how common phenotypic behaviors, such as PDGF receptor-driven gliomagenesis, can arise from a variety of genomic events. From a therapeutic standpoint, the similarities in the biology of the members of these tumor subtypes may outweigh differences in the details of how the pathways are driven, allowing this class of gliomas to respond similarly to therapeutic intervention.

\section{Materials and methods}

Glioma specimens

Formalin-fixed paraffin-embedded (FFPE) and frozen archival human specimens were obtained from the following institutions: 
Kanazawa University, Kanazawa, Japan (KNZ; 53 cases); Kitasato University, Kanagawa, Japan (KTS; 56 cases); Kobe University, Hyogo, Japan (KB; 48 cases); Memorial Sloan-Kettering Cancer Center, New York, NY (MSK; 58 cases, including three tumorderived cell lines). Pathological diagnosis of these tumors was done according to the WHO classification at individual institutions. A summary of the tumor samples is described in Supplemental Fig. S2. Although MSK538 was diagnosed as an anaplastic astrocytoma in the report from the pathology department, the tumor was classified as a GBM in this study by consideration of the clinical course and molecular profiles. Both DNA and RNA from MSK543 and MSK573 were extracted from individual tumorderived cell lines, respectively. DNA from MSK667 was extracted from the tumor-derived cell line as well.

\section{aCGH analysis and identification of the KP fusion transcript}

aCGH detection and mapping of intragenic copy number aberration (iCNA) was performed in two stages. A screening array was designed to cover 98 TKs, each with 75 oligonucleotide probes of 60 -bp length directed to three regions: the $5^{\prime}$ coding region, the coding region $3^{\prime}$ to the kinase domain, and the $5^{\prime}$ promoter region (Agilent Technologies). Candidate iCNA events were detected as a shift in $\log 2$ ratio between the $5^{\prime}$ and $3^{\prime}$ probe sets (Student's $t$-test). The paired iCNA events in KDR and PDGFRA were verified and fine-mapped on a second array with tiling coverage through the PDGFRA-KIT-KDR locus: 12,487 probes covering $54.92-55.85 \mathrm{Mb}$ on chromosome 4 with a median interprobe interval of 48 bp and 2874 reverse-complement replicate oligonucleotide probes. Probes were optimized for Tm by adjusting the length (45-60mer). For breakpoint mapping, the tumor profile was normalized by control profiles from tumors with uniform amplification through the PDGFRA and KDR genes in order to account for variation in individual probe performance (nonspecific hybridization). All results are presented using human genome build hg18.

A $4 \times 3$-matrix RT-PCR panel was designed to analyze all of the possible in-frame exon combinations between KDR and PDGFRA around their potential intragenic breakpoint regions. Information about forward primers in KDR exons 3, 6, 9, and 13 and reverse primers in PDGFRA exons 7, 9, and 12 is listed in the Supplemental Material. RT-PCR was performed using the Qiagen one-step RT-PCR kit. PCR products were electrophoresed on agarose gels. The promising RT-PCR products were purified with a Qiagen gel purification kit and sequenced on an Applied Biosystem automated sequencer. RT-PCR screening of the KP fusion was performed mainly with the K13/P12 primer pair. A KDR-S and PDGFRA-AS2 primer pair was also partly used.

\section{Screening of the PDGFRA ${ }^{\Delta 8,9}$ mutant}

The identification of the PDGFRA ${ }^{\Delta 8,9}$ mutant was performed individually with RT-PCR analysis using a total of 212 glioma tumors at four institutions. Total RNAs were isolated with the RNeasy Lipid Tissue Minikit (Qiagen), and were used to synthesize cDNAs by using the SuperScript system (Invitrogen) according to the manufacturer's protocol. The PDGFRA-S3/AS2 or A/D primer pairs were used for the detection of wild-type PDGFRA and the PDGFRA ${ }^{\Delta 8,9}$ mutant. The sequences of the A and D primers were described previously (Kumabe et al. 1992). PCR amplification was performed with Taq DNA polymerase (Invitrogen), and the protocol was $3 \mathrm{~min}$ at $94^{\circ} \mathrm{C}$, denaturation for 45 sec at $94^{\circ} \mathrm{C}$, annealing for $30 \mathrm{sec}$ at $55^{\circ} \mathrm{C}$, and extension for $90 \mathrm{sec}$ at $72^{\circ} \mathrm{C}$ for 30 cycles, followed by $10 \mathrm{~min}$ of final extension. The
PCR products were resolved by electrophoresis on $2 \%$ agarose gels for S3/AS2 or 3\% agarose gels for A/D primer pair with ethidium bromide. RNA extraction and RT-PCR were performed with the protocol partially modified at individual institutions. The RT-PCR analysis at Kitasato University was partly performed by T.O. The promising RT-PCR products were purified with the Qiagen gel purification kit and sequenced with PDGFRA-S7 or PDGFRA-S8 primers on an Applied Biosystem automated sequencer, or were cloned into pGEM-T vector (Promega) and then sequenced as well. The information about the primers is listed in the Supplemental Material.

PDGFRA gene status was determined by q-PCR analysis in the 87 tumors with significant PDGFRA mRNA overexpression, which was examined with the PDGFRA-S3/AS2 primer pair. MSK259 and KNZ15 were excluded from this analysis because no DNA samples were available. DNA was extracted from human frozen brain tumor tissues or paraffin sections using the DNeasy Blood and Tissue Kit (Qiagen) and quantitated on a NanoDrop spectrophotometer (Thermo Scientific). Selected areas containing at least $85 \%$ of the tumor were macroscopically and manually dissected from paraffin sections. Reference DNA was obtained from lymphocytes from a total of four healthy volunteers. q-PCR was performed in triplicate using each PDGFRA and $18 S$ gene-specific primer set as described previously (Martinho et al. 2009), reagents, and protocols from Applied Biosystems in a 7900 HT Fast Real-Time PCR System. The PCR amplification was performed in a $10-\mu \mathrm{L}$ reaction volume under the following conditions: $2 \times$ Power SYBR Green PCR Master Mix (Applied Biosystems), $0.5 \mu \mathrm{M}$ primers, and 20 ng of DNA. The $\triangle \Delta \mathrm{Ct}$ method was used to calculate PDGFRA gene copy number in the tumor DNA sample normalized to the reference gene $(18 S)$ and calibrated to normal DNA. Gene amplification was defined as average DNA quantities greater than four, corresponding to more than eight copies.

\section{Statistical analysis}

All values are expressed as means \pm SD. Statistical significance was determined using a two-tailed Student's $t$-test using GraphPad Prism software, and a value of $P<0.05$ was considered significant. For the analysis of tumor growth on nude mice, $P$-values were determined at the final point.

\section{Acknowledgments}

We thank Jim Finney and Qunchao Zhang for technical assistance, Eletha Carbajal and Charlene Williams for secretarial assistance, and the Research Animal Resource Center for mouse maintenance. We are also grateful to Dr. Hideyuki Saya in Keio University for suggestions. This work was supported by NIH grant UO1CA04002. T.O. was supported by Susan Kramer/ American Brain Tumor Association Fellowship. C.B. was supported in part by Accelerate Brain Cancer Cure (ABC2).

\section{References}

Blume-Jensen P, Hunter T. 2001. Oncogenic kinase signalling. Nature 411: 355-365.

Brennan C, Momota H, Hambardzumyan D, Ozawa T, Tandon A, Pedraza A, Holland E. 2009. Glioblastoma subclasses can be defined by activity among signal transduction pathways and associated genomic alterations. PLOS ONE 4: e7752. doi: 10.1371/journal.pone.0007752.

Campbell PJ, Stephens PJ, Pleasance ED, O'Meara S, Li H, Santarius T, Stebbings LA, Leroy C, Edkins S, Hardy C, 
et al. 2008. Identification of somatically acquired rearrangements in cancer using genome-wide massively parallel paired-end sequencing. Nat Genet 40: 722-729.

The Cancer Genome Atlas Research Network. 2008. Comprehensive genomic characterization defines human glioblastoma genes and core pathways. Nature 455: 1061-1068.

Charest A, Lane K, McMahon K, Park J, Preisinger E, Conroy H, Housman D. 2003. Fusion of FIG to the receptor tyrosine kinase ROS in a glioblastoma with an interstitial del(6)(q21q21). Genes Chromosomes Cancer 37: 58-71.

Chu CT, Everiss KD, Wikstrand CJ, Batra SK, Kung HJ, Bigner DD. 1997. Receptor dimerization is not a factor in the signalling activity of a transforming variant epidermal growth factor receptor (EGFRvIII). Biochem I 324: 855-861.

Clarke ID, Dirks PB. 2003. A human brain tumor-derived PDGFR$\alpha$ deletion mutant is transforming. Oncogene 22: 722-733.

Dai C, Celestino JC, Okada Y, Louis DN, Fuller GN, Holland EC. 2001. PDGF autocrine stimulation dedifferentiates cultured astrocytes and induces oligodendrogliomas and oligoastrocytomas from neural progenitors and astrocytes in vivo. Genes Dev 15: 1913-1925.

Druker BJ, Tamura S, Buchdunger E, Ohno S, Segal GM, Fanning S, Zimmermann J, Lydon NB. 1996. Effects of a selective inhibitor of the Abl tyrosine kinase on the growth of Bcr-Abl positive cells. Nat Med 2: 561-566.

Ekstrand AJ, James CD, Cavenee WK, Seliger B, Pettersson RF, Collins VP. 1991. Genes for epidermal growth factor receptor, transforming growth factor $\alpha$, and epidermal growth factor and their expression in human gliomas in vivo. Cancer Res 51: 2164-2172.

Faraone D, Aguzzi MS, Ragone G, Russo K, Capogrossi MC, Facchiano A. 2006. Heterodimerization of FGF-receptor 1 and PDGF-receptor- $\alpha$ : A novel mechanism underlying the inhibitory effect of PDGF-BB on FGF-2 in human cells. Blood 107: 1896-1902.

Gan HK, Kaye AH, Luwor RB. 2009. The EGFRvIII variant in glioblastoma multiforme. I Clin Neurosci 16: 748-754.

Holland EC, Hively WP, DePinho RA, Varmus HE. 1998. A constitutively active epidermal growth factor receptor cooperates with disruption of G1 cell-cycle arrest pathways to induce glioma-like lesions in mice. Genes Dev 12: 36753685.

Homma T, Fukushima T, Vaccarella S, Yonekawa Y, Di Patre PL, Franceschi S, Ohgaki H. 2006. Correlation among pathology, genotype, and patient outcomes in glioblastoma. I Neuropathol Exp Neurol 65: 846-854.

Jones DT, Kocialkowski S, Liu L, Pearson DM, Backlund LM, Ichimura K, Collins VP. 2008. Tandem duplication producing a novel oncogenic BRAF fusion gene defines the majority of pilocytic astrocytomas. Cancer Res 68: 8673-8677.

Jones DT, Kocialkowski S, Liu L, Pearson DM, Ichimura K, Collins VP. 2009. Oncogenic RAF1 rearrangement and a novel BRAF mutation as alternatives to KIAA1549:BRAF fusion in activating the MAPK pathway in pilocytic astrocytoma. Oncogene 28: 2119-2123.

Kumabe T, Sohma Y, Kayama T, Yoshimoto T, Yamamoto T. 1992. Amplification of $\alpha$-platelet-derived growth factor receptor gene lacking an exon coding for a portion of the extracellular region in a primary brain tumor of glial origin. Oncogene 7: 627-633.

Kumar-Sinha C, Tomlins SA, Chinnaiyan AM. 2006. Evidence of recurrent gene fusions in common epithelial tumors. Trends Mol Med 12: 529-536.

Martinho O, Longatto-Filho A, Lambros MB, Martins A, Pinheiro C, Silva A, Pardal F, Amorim J, Mackay A, Milanezi F, et al. 2009. Expression, mutation and copy number analysis of platelet-derived growth factor receptor A (PDGFRA) and its ligand PDGFA in gliomas. Br J Cancer 101: 973-982.

Mellinghoff IK, Wang MY, Vivanco I, Haas-Kogan DA, Zhu S, Dia EQ, Lu KV, Yoshimoto K, Huang JH, Chute DJ, et al. 2005. Molecular determinants of the response of glioblastomas to EGFR kinase inhibitors. N Engl J Med 353: 20122024.

Mitelman F, Johansson B, Mertens F. 2004. Fusion genes and rearranged genes as a linear function of chromosome aberrations in cancer. Nat Genet 36: 331-334.

Mitelman F, Johansson B, Mertens F. 2007. The impact of translocations and gene fusions on cancer causation. Nat Rev Cancer 7: 233-245.

Ohgaki H, Kleihues P. 2007. Genetic pathways to primary and secondary glioblastoma. Am J Pathol 170: 1445-1453.

Phillips HS, Kharbanda S, Chen R, Forrest WF, Soriano RH, Wu TD, Misra A, Nigro JM, Colman H, Soroceanu L, et al. 2006. Molecular subclasses of high-grade glioma predict prognosis, delineate a pattern of disease progression, and resemble stages in neurogenesis. Cancer Cell 9: 157-173.

Rand V, Huang J, Stockwell T, Ferriera S, Buzko O, Levy S, Busam D, Li K, Edwards JB, Eberhart C, et al. 2005. Sequence survey of receptor tyrosine kinases reveals mutations in glioblastomas. Proc Natl Acad Sci 102: 14344-14349.

Rupp E, Siegbahn A, Ronnstrand L, Wernstedt C, ClaessonWelsh L, Heldin CH. 1994. A unique autophosphorylation site in the platelet-derived growth factor $\alpha$ receptor from a heterodimeric receptor complex. Eur I Biochem 225: 29-41.

Saito Y, Haendeler J, Hojo Y, Yamamoto K, Berk BC. 2001. Receptor heterodimerization: Essential mechanism for platelet-derived growth factor-induced epidermal growth factor receptor transactivation. Mol Cell Biol 21: 6387-6394.

Stommel JM, Kimmelman AC, Ying H, Nabioullin R, Ponugoti $\mathrm{AH}$, Wiedemeyer $\mathrm{R}$, Stegh $\mathrm{AH}$, Bradner JE, Ligon KL, Brennan C, et al. 2007. Coactivation of receptor tyrosine kinases affects the response of tumor cells to targeted therapies. Science 318: 287-290.

Sugawa N, Ekstrand AJ, James CD, Collins VP. 1990. Identical splicing of aberrant epidermal growth factor receptor transcripts from amplified rearranged genes in human glioblastomas. Proc Natl Acad Sci 87: 8602-8606.

Tao Q, Backer MV, Backer JM, Terman BI. 2001. Kinase insert domain receptor (KDR) extracellular immunoglobulin-like domains 4-7 contain structural features that block receptor dimerization and vascular endothelial growth factor-induced signaling. J Biol Chem 276: 21916-21923.

Tomlins SA, Rhodes DR, Perner S, Dhanasekaran SM, Mehra R, Sun XW, Varambally S, Cao X, Tchinda J, Kuefer R, et al. 2005. Recurrent fusion of TMPRSS2 and ETS transcription factor genes in prostate cancer. Science 310: 644-648.

Uhrbom L, Hesselager G, Nister M, Westermark B. 1998. Induction of brain tumors in mice using a recombinant platelet-derived growth factor B-chain retrovirus. Cancer Res 58: 5275-5279.

Verhaak RG, Hoadley KA, Purdom E, Wang V, Qi Y, Wilkerson MD, Miller CR, Ding L, Golub T, Mesirov JP, et al. 2010. Integrated genomic analysis identifies clinically relevant subtypes of glioblastoma characterized by abnormalities in PDGFRA, IDH1, EGFR, and NF1. Cancer Cell 17: 98-110.

Wen PY, Yung WK, Lamborn KR, Dahia PL, Wang Y, Peng B, Abrey LE, Raizer J, Cloughesy TF, Fink K, et al. 2006. Phase I/II study of imatinib mesylate for recurrent malignant gliomas: North American Brain Tumor Consortium Study 99-08. Clin Cancer Res 12: 4899-4907. 
Ozawa et al.

Wong AJ, Ruppert JM, Bigner SH, Grzeschik CH, Humphrey PA, Bigner DS, Vogelstein B. 1992. Structural alterations of the epidermal growth factor receptor gene in human gliomas. Proc Natl Acad Sci 89: 2965-2969.

Wood JM, Bold G, Buchdunger E, Cozens R, Ferrari S, Frei J, Hofmann F, Mestan J, Mett H, O'Reilly T, et al. 2000. PTK787/ZK 222584, a novel and potent inhibitor of vascular endothelial growth factor receptor tyrosine kinases, impairs vascular endothelial growth factor-induced responses and tumor growth after oral administration. Cancer Res 60: 2178-2189.

Zhu H, Acquaviva J, Ramachandran P, Boskovitz A, Woolfenden S, Pfannl R, Bronson RT, Chen JW, Weissleder R, Housman DE, et al. 2009. Oncogenic EGFR signaling cooperates with loss of tumor suppressor gene functions in gliomagenesis. Proc Natl Acad Sci 106: 2712-2716. 


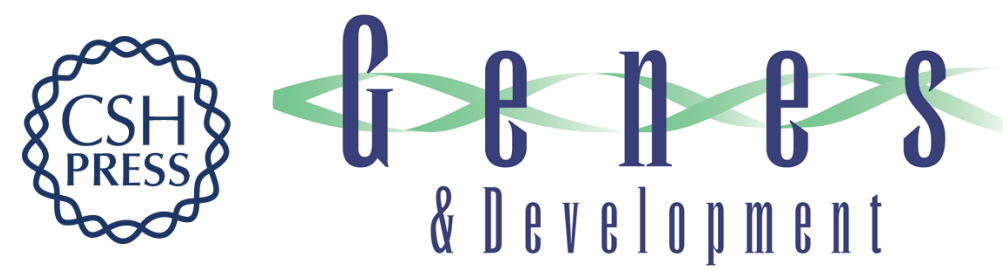

\section{PDGFRA gene rearrangements are frequent genetic events in PDGFRA -amplified glioblastomas}

Tatsuya Ozawa, Cameron W. Brennan, Lu Wang, et al.

Genes Dev. 2010, 24:

Access the most recent version at doi:10.1101/gad.1972310

Supplemental http://genesdev.cshlp.org/content/suppl/2010/09/27/24.19.2205.DC1
Material

References This article cites 37 articles, 17 of which can be accessed free at: http://genesdev.cshlp.org/content/24/19/2205.full.html\#ref-list-1

License

Email Alerting

Receive free email alerts when new articles cite this article - sign up in the box at the top Service right corner of the article or click here.

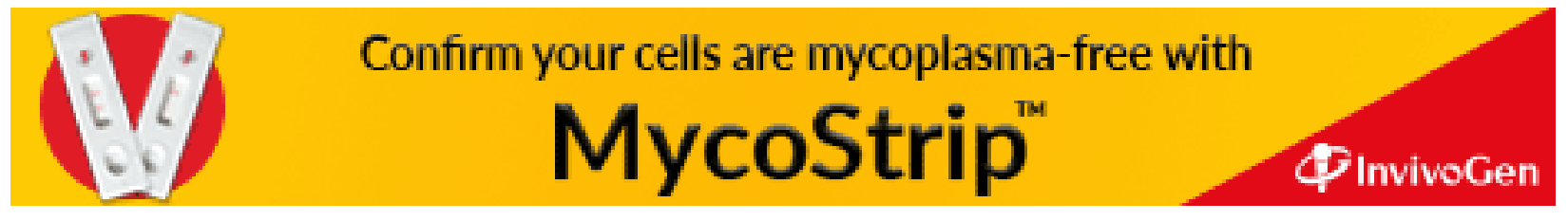

